Original Article (short paper)

\title{
Is a short-stage protocol during an incremental exercise test reliable for heart rate variability threshold analysis?
}

\author{
Carlos Janssen Gomes da Cruz ${ }^{1,2}$, Luiz Guilherme Grossi Porto²®, Giliard Lago Garcia² ${ }^{2}$, \\ Renato André Sousa da Silva ${ }^{1}\left[\right.$, Guilherme Eckhardt Molina ${ }^{2}{ }^{\circledR}$ \\ ${ }^{I}$ Centro Universitário Euro-Americano, Departamento de Educação Física, Grupo de Estudos e \\ Pesquisas em Função Autonômica Cardíaca, GEFAC, Brasilia, DF, Brasil; ' ${ }^{2}$ Universidade de Brasília, \\ Faculdade de Educação Física, Laboratório de Fisiologia do Exercício, Brasilia, DF, Brasil
}

\begin{abstract}
Aim: Heart rate variability threshold (HRVT) is a valid method to determine parasympathetic depression during an incremental exercise test (IET). However, $\mathrm{HRV}_{\mathrm{T}}$ is usually assessed using the last $60 \mathrm{~s}$ of each $180 \mathrm{~s}$ stage of an IET, resulting in longer and demotivating tests. This study aimed to evaluate the agreement of HRV т analysis adopting the first and second minute of R-R interval (iRR) segment comparatively to a standard third-minute segment obtained at each 3-min stage on IET. Methods: Seventeen young male subjects $\left(22.2 \pm 3.1\right.$ years; $\left.23.4 \pm 2.3 \mathrm{~kg} / \mathrm{m}^{2}\right)$ underwent IET on a cycle ergometer. $\mathrm{HRV}_{\text {т }}$ was considered the load corresponding to the point of stabilization of the SD1 index $\left(\mathrm{HRV}_{\mathrm{Tv}}\right)$, or the first load with SD1 value $<3 \mathrm{~ms}\left(\mathrm{HRV}_{\mathrm{T}<3}\right)$, both assessed by the $1^{\text {st }}\left(\mathrm{HRV}_{\mathrm{T} 1 \mathrm{~V}}, \mathrm{HRV}_{\mathrm{T} 1<3}\right), 2^{\text {nd }}\left(\mathrm{HRV}_{\mathrm{T} 2 \mathrm{~V}}\right.$, $\left.\mathrm{HRV}_{\mathrm{T2}<3}\right)$ and standard $3^{\text {rd }}\left(\mathrm{HRV}_{\mathrm{T} 3 \mathrm{~V}}, \mathrm{HRV} \mathrm{TR}_{3}<3\right) 60 \mathrm{~s}$ iRR segment analyzed at each stage of IET. Agreement and reliability were assessed by the Bland-Altman analysis and the intraclass correlation coefficient (ICC), respectively. Results: High reliability and non-significant bias were observed considering $\mathrm{HRV}_{\mathrm{TIV}}$ vs $\mathrm{HRV}_{\mathrm{T} 3 \mathrm{~V}}(\mathrm{ICC}=0.92 ; p=0.18)$ or $\mathrm{HRV}_{\mathrm{T} 2 \mathrm{~V}} v s$ HRVтзи $(\mathrm{ICC}=0.94 ; p=0.99)$. However, lower reliability was observed for $\mathrm{HRV}_{\mathrm{T} 1<3} v s \mathrm{HRV} \mathrm{H}_{\mathrm{T}<3}(\mathrm{ICC}=0.79 ; p=0.75)$ and for HRV т2<3 vs HRV тз<3 $(\mathrm{ICC}=0.91 ; p=0.33)$. Conclusion: HRVt can be similarly assessed by the $1^{\text {st }}, 2^{\text {nd }}$ or $3^{\text {rd }}$ 60 seconds iRR segment, mainly when assessed by a visual method.
\end{abstract}

Keywords: heart rate variability, anaerobic threshold, exercise test, parasympathetic nervous system, cardiac autonomic function.

\section{Introduction}

Heart rate variability (HRV) consists of the fluctuations in R-R interval (iRR) between successive heartbeats defined by the distance between two R-waves on the electrocardiogram ${ }^{1,2}$. HRV has been used either in sports or in clinical settings as a proxy of the cardiac autonomic function ${ }^{3,4}$. In this sense, different HRV methods and indices have been proposed to evaluate the parasympathetic activation or its withdraw associated with various physiological conditions such as exercise ${ }^{5,6}$.

During an incremental exercise test (IET), an exponential reduction of the Poincaré map - SD1, a parasympathetic index of $\mathrm{HRV}$, is expected as a consequence of parasympathetic activity reduction during exercise ${ }^{5}$. Hence, the point where the values of $\mathrm{SD} 1$ is stabilized is called heart rate variability threshold (HRVT) and it is considered an indicator of the parasympathetic activity plateau during the IET ${ }^{5,7}$.

In addition to reflecting the parasympathetic plateau during exercise, some researchers showed that HRV coincides with lactate (LT) and first ventilatory (VT) thresholds ${ }^{8-12}$. Thus, the analysis of HRV and low-cost strategy for the anaerobic threshold (AT) determination ${ }^{8,12}$.
Although the physiological interpretation of these thresholds is not consensual, the determination of these markers is an efficient strategy for endurance performance diagnostic and prognostic and is widely used for better control and prescription of the cardiorespiratory training intensity ${ }^{14,15}$. In this scenario, considering the different methods traditionally used to estimate the anaerobic threshold (LT, VT, and HRVT), $\mathrm{HRV}_{\mathrm{T}}$ determination stands out due to the low-cost analysis and the non-laboratory or non-invasive procedure. However, most studies that validated the use of HRVT to estimate LT usually apply incremental exercise protocols with stages of 3 minutes of duration ${ }^{11,12,16}$, which results in longer and demotivating tests and low values of maximal power output compared with short stages protocols ${ }^{17}$.

Of note, the rationale for the large stage protocol (3-min) approach to determine the LT and the $\mathrm{HRV}_{\mathrm{T}}$ is the period necessary for the muscle lactate to be available in the bloodstream and for iRR stabilization, respectively ${ }^{11,15}$. However, the HRVT is dependent on cardiac autonomic adjustments, which are fast neural adaptations that precede the blood lactate increase. Therefore, it is reasonable to question the necessity to adopt large stage protocols (3 minutes) during IET aiming the HRVT determination. 
From that perspective, this study aimed to evaluate the agreement and reliability of $\mathrm{HRV}_{\mathrm{T}}$ determination by 60 seconds iRR segments obtained from the first $\left(\mathrm{HRV}_{\mathrm{T} 1}\right)$ and second (HRV 2 ) minutes comparatively to the standard 3rd (HRV T3) minute segment of each IET stage among healthy and physically active male adults. Due to the moderate to high interday reliability of HRV T assessed in short-stage protocols ${ }^{13,18}$, we hypothesize that $\mathrm{HRV}_{\mathrm{T}}$ can be similarly assessed by the first, second or even in the third iRR segment of analysis during an incremental exercise test.

\section{Methods}

\section{Participants}

We evaluated seventeen physically active young men ( $22.2 \pm 2.1$ years). The sample size was defined considering an effect size of $0.35(f)$ and a Power of $0.80(1-\beta)$. As inclusion criteria, the participants had to be between 20 to 30 years old, be nonsmokers, with no participation in competitive sports, without any reported clinical disease, symptomatic clinical manifestations, muscle or joint disorders, or be taking medications. Those who started drug treatment during the data collection period or present excessive artifact beats in iRR segments $(>2 \%)$ were excluded $(n=2)^{19}$.

To avoid possible circadian influences on HRV dynamics ${ }^{20}$, all participants underwent an exercise testing between 8:00 and 11:00 am. Previously, they had been instructed to abstain from stimulants, alcoholic beverages, medicines and physical activity for at least $48 \mathrm{~h}$ before exercise testing. The volunteers were informed about the experimental protocol before beginning all tests, and all signed an informed consent to participate, with the approval of the Ethical Committee on Human Research of the Centro Universitário Euro-Americano - UNIEURO (protocol number: 2.320.164), in compliance with the Brazilian National Research Ethics System Guidelines and the Declaration of Helsinki.

Initially, we obtained clinical and anthropometrical (body mass and height) data, basic lifestyle habits, as well as smoking and alcohol consumption habits, symptoms and/or diagnoses of chronic diseases and physical activity level. The physical activity level was assessed using the International Physical Activity Questionnaire-IPAQ ${ }^{21}$ and all other information were obtained by a clinical anamnesis. Resting heart rate (HR) and blood pressure were measured after five minutes of resting in the sitting position using the V800 Polar $^{\circledR}$ HR monitor and the auscultatory method, respectively. All measures were performed in a quiet clinical laboratory room at ambient temperature $\left(21^{\circ} \mathrm{C}-24^{\circ} \mathrm{C}\right)$.

\section{Incremental Exercise Testing}

The incremental exercise testing was performed in an electro-magnetically braked cycle ergometer (Movement ${ }^{\circledR} 3500$ Pro, Brazil). The submaximal exercise testing was initiated with a load of 25 Watts (W) and an increment of $25 \mathrm{~W}$ was adopted every 3 minutes ${ }^{11}$ until the volunteer reached $85 \%$ of his maximum predicted heart rate $\left(\mathrm{HR}_{\max }\right)$. The $\mathrm{HR}_{\max }$ was predicted by the formula $H_{\max }=208-(0.7 \mathrm{x} \text { age })^{22}$. During the exercise testing, the volunteers were oriented to maintain a cycling speed of 60-70 revolutions per minute.

\section{Heart Rate Variability Threshold Determination}

A cardiac monitor $\left(\right.$ Polar $^{\circledR}$ V800) was used to record iRR series during the IET $^{23-25}$. Then, each series was transferred to a microcomputer for offline data processing and analysis of iRR variability employing software Kubios HRV analyzer ${ }^{\circledR}$ version 2.0 (Kuopio, Finland) ${ }^{26}$. Before processing the HRV data, all iRR series were visually verified on a beat-to-beat basis to identify ectopic beats and artifacts. When present, spurious beats were deleted from the series without adding new intervals which accounted for less than $2 \%$ in every subject ${ }^{19}$.

To determine the HRV with 3 minutes of duration in each stage. During each stage, SD1 index of HRV was analyzed at three different time points: a) first minute (0-60s), b) second minute (60-120s) and c) third minute (120-180s). HRV T was assessed on each 1-min period (HRVT1, $\mathrm{HRV}_{\mathrm{T} 2}$ and $\mathrm{HRV}_{\mathrm{T} 3}$, respectively), as described in Figure 1a.

$\mathrm{HRV}_{\mathrm{T}}$ was assessed adopting two methods a) the load (W) corresponding to the point of stabilization in which there was no further significant decline in the values of the SD1 during IET, identified by means of visual graphic evaluation (visual method-HRV $\left.{ }_{\text {TV }}\right)^{9,13,27}$; b) the first load with SD1 value lower than $3 \mathrm{~ms}$ (mathematical method-HRV $\mathrm{T}<3)^{7}$. Both methods are illustrated in the Figure $1 \mathrm{~b}$.

$\mathrm{HRV}_{\mathrm{T}}$ analysis was performed by two independent experienced evaluators. In case of disagreement between them, a third evaluator would be consulted to examine the graph. All data were analyzed by consensus between the two evaluators, thus, the third evaluator was not necessary.

To evaluate the possible influence of stage duration on stability of HR and HRV during different phases of IET, we calculated HR, SD1, standard deviation of iRR (SDNN) and coefficient of variation (CV) in the first, second and third 60s segments of the iRR series obtained in the three-time points during exercise: 1) the first stage of IET, 2) the load corresponding to $\mathrm{HRV}_{\mathrm{TV}}$ and $\mathrm{HRV}_{\mathrm{T}<3}$; and 3 ) the last stage of IET. CV is a measure of discrepancy and it is expressed as a percentage of the mean $\mathrm{iRR}(\mathrm{CV}=$ standard deviation of $\mathrm{iRR} /$ mean $\mathrm{iRR} \times 100)$.

\section{Statistical Analyses}

The normality of the data was confirmed by the ShapiroWilk test. Comparison between the three segments was made using ANOVA for repeated measures considering sphericity correction by Greenhouse-Geisser method. The differences between conditions were identified by Bonferroni post-hoc test and effect size was represented by partial eta squared $\left(\eta_{p}{ }^{2}\right)$.

Agreement and reliability between analysis were performed according to the Bland-Altman analysis ${ }^{28}$ and the intraclass correlation coefficient $(\mathrm{ICC})^{29}$, respectively. A one-sample 
t-test was used to test the hypothesis that the bias between measurements was zero. The results were considered statistically significant when the probability associated with the type I error was less than or equal to $5 \%(\mathrm{p}<0.05)$. Statistical analysis of the data was performed using SPSS v.20 (SPSS Inc., USA).

\section{Results}

The basic resting physiological variables and anthropometrical characteristics of participants are shown in Table 01. According to IPAQ, the participants presented moderate (62\%) to high (38\%) physical activity level.
Table 1. Mean and standard deviation (SD) of anthropometrical and resting physiological data

\begin{tabular}{|c|c|}
\hline Variables & Mean (SD) \\
\hline Body mass (kilograms) & $71.9 \pm 7.8$ \\
\hline Height (centimeters) & $175.1 \pm 6.9$ \\
\hline BMI $\left(\mathrm{kg} / \mathrm{m}^{2}\right)$ & $23.4 \pm 2.3$ \\
\hline HR (bpm) & $78.4 \pm 7.8$ \\
\hline $\mathrm{SBP}(\mathrm{mmHg})$ & $117.6 \pm 8.1$ \\
\hline $\mathrm{DBP}(\mathrm{mmHg})$ & $74.6 \pm 7.7$ \\
\hline
\end{tabular}

$\mathrm{BMI}=$ body mass index (kilograms per square meter), $\mathrm{HR}=$ heart rate (beats per minute), $\mathrm{SBP}=$ systolic blood pressure (millimeters of mercury), DBP = diastolic blood pressure (millimeters of mercury).

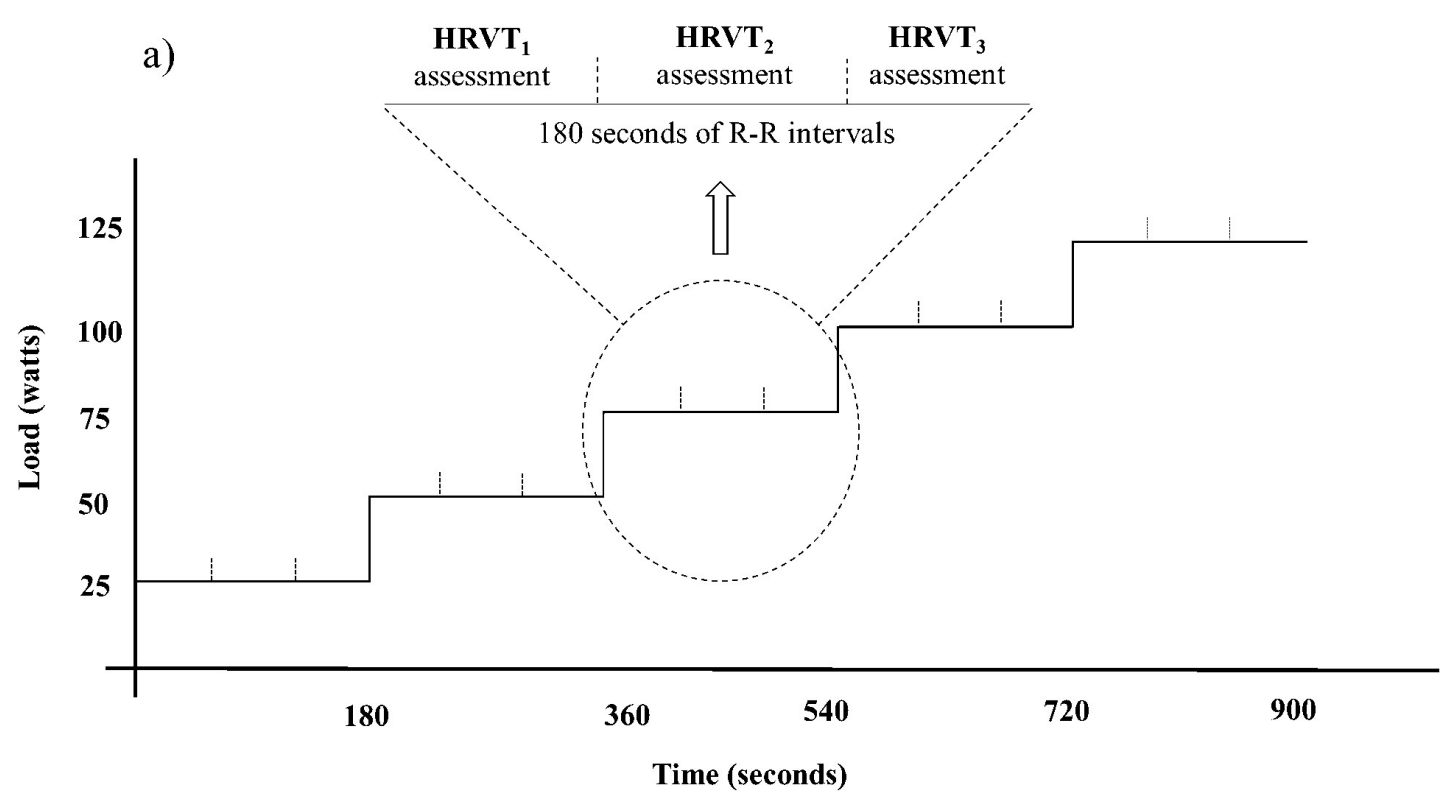

b)

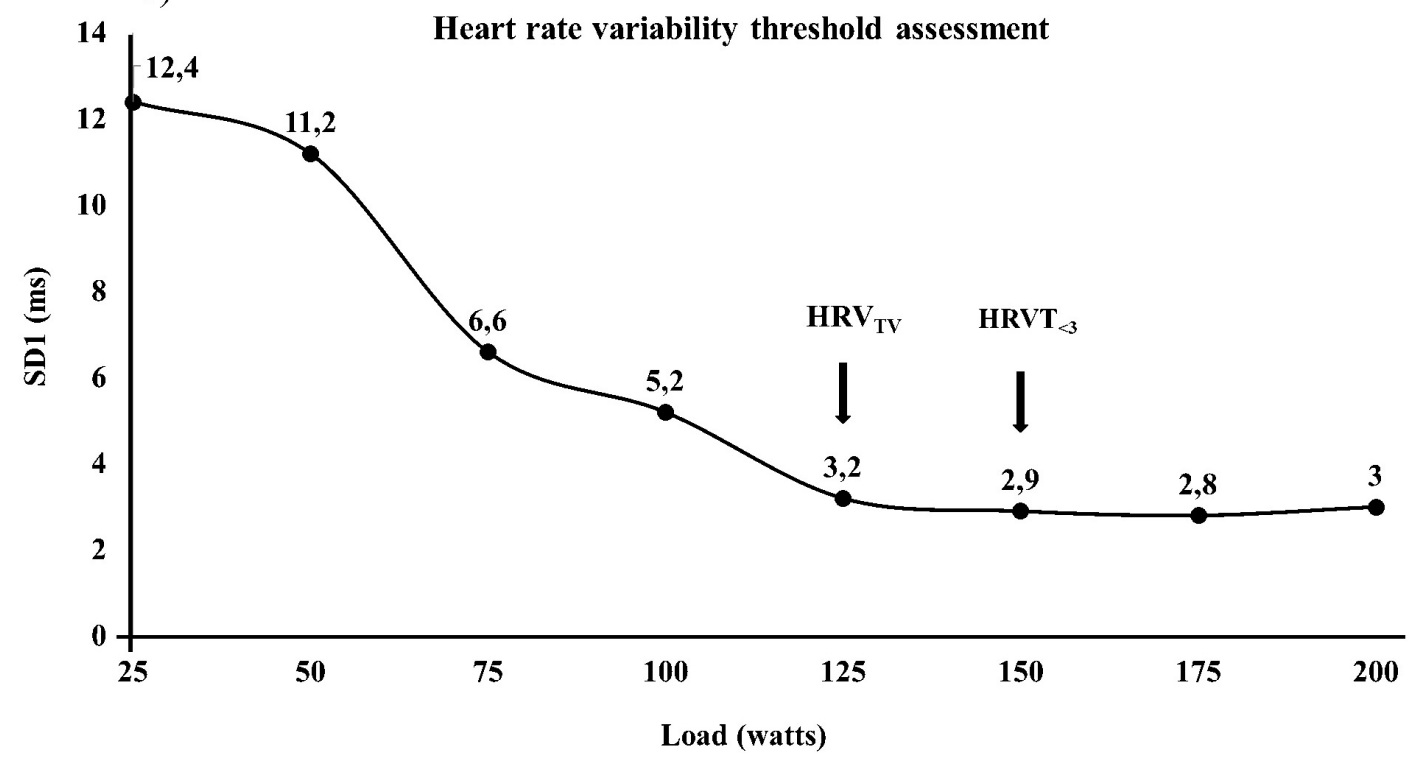

Figure 1. a) Schematic illustration of heart rate variability analysis in each stage of the incremental exercise test. b) Representation of heart rate variability threshold assessed by visual and mathematical methods. 
No differences were observed between HR assessed at first, second and third minute in the initial stage of exercise test $\left(p=0.21, \eta_{\mathrm{p}}{ }^{2}=0.17\right)$ or in the stage corresponding to HRVT<3 $\left(p=0.81, \eta_{\mathrm{p}}{ }^{2}=0.01\right)$. On the other hand, low HR was observed for HRVTV1 compared to $\operatorname{HRV}_{\mathrm{TV} 3}\left(p=0.04, \eta_{\mathrm{p}}{ }^{2}=0.19\right)$ and for HR assessed in the first and second minute compared to the third minute in the last stage of IET $\left(p<0.001, \eta_{p}{ }^{2}=0.78\right)$ (Figure 2a).

Considering HRV analysis, no differences were identified between the three segments of analysis for SDNN (Figure 2b), SD1 (Figure 2c) and CV (Figure 2d) indices assessed in the initial stage of IET (SDNN: $p=0.78, \eta_{\mathrm{p}}^{2}=0.01, \mathrm{SD} 1: p=0.48$, $\left.\eta_{\mathrm{p}}{ }^{2}=0.04, \mathrm{CV}: p=0.67, \eta_{\mathrm{p}}{ }^{2}=0.02\right)$, neither in the stages corresponding to HRVTV (SDNN: $p=0.98, \eta_{\mathrm{p}}{ }^{2}=0.001$, SD1: $\left.p=0.12, \eta_{\mathrm{p}}^{2}=0.13, \mathrm{CV}: p=0.79, \eta_{\mathrm{p}}^{2}=0.01\right)$ and $\mathrm{HRV}_{\mathrm{T}<3}(\mathrm{SDNN}$ : $p=0.63, \eta_{\mathrm{p}}{ }^{2}=0.02, \mathrm{SD} 1: p=0.54, \eta_{\mathrm{p}}{ }^{2}=0.03, \mathrm{CV}: p=0.71$, $\left.\eta_{\mathrm{p}}{ }^{2}=0.01\right)$ nor in the least stage of exercise test (SDNN: $p=0.23$, $\left.\eta_{\mathrm{p}}{ }^{2}=0.09, \mathrm{SD} 1: p=0.54, \eta_{\mathrm{p}}{ }^{2}=0.03, \mathrm{CV}: p=0.43, \eta_{\mathrm{p}}{ }^{2}=0.04\right)$.

Similarly, no differences were observed between the load (watts) corresponding to HRVT1V $(106.1 \pm 23.2)$, HRV T2V $(101.1 \pm 28.1)$ and HRV $_{\mathrm{T} 3 \mathrm{~V}}(106.1 \pm 25.5)\left(p=0.18, \eta_{\mathrm{p}}{ }^{2}=0.11\right)$ or between $\mathrm{HRV}_{\mathrm{T} 1<3}(98.4 \pm 19.2), \mathrm{HRV}_{\mathrm{T} 2<3}(98.4 \pm 28.1)$ and $\operatorname{HRV}_{\mathrm{T} 3<3}(96.8 \pm 27.1)\left(p=0.79, \eta_{\mathrm{p}}^{2}<0.001\right)$. Additionally, we observed higher ICC and a non-significant bias between $\mathrm{HRV}_{\mathrm{T} 1 \mathrm{v}}$ vs HRV T3v $(\mathrm{ICC}=0.92)$ and between HRV 2 v vs HRV T3V $(\mathrm{ICC}=0.94)$. However, lower ICC values were observed between $\mathrm{HRV}_{\mathrm{T} 1<3}$ vs $\mathrm{HRV}_{\mathrm{T} 3<3}(\mathrm{ICC}=0.79)($ Figures 3a-b) and between HRVT2 $<3$ vs HRVт3 $<3(\mathrm{ICC}=0.91)$ (Figures 3c-d). a)

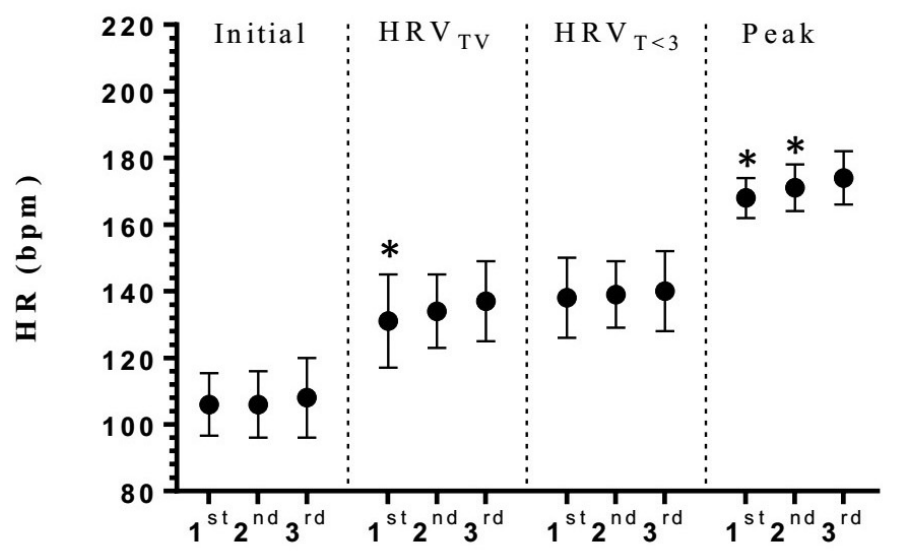

c)

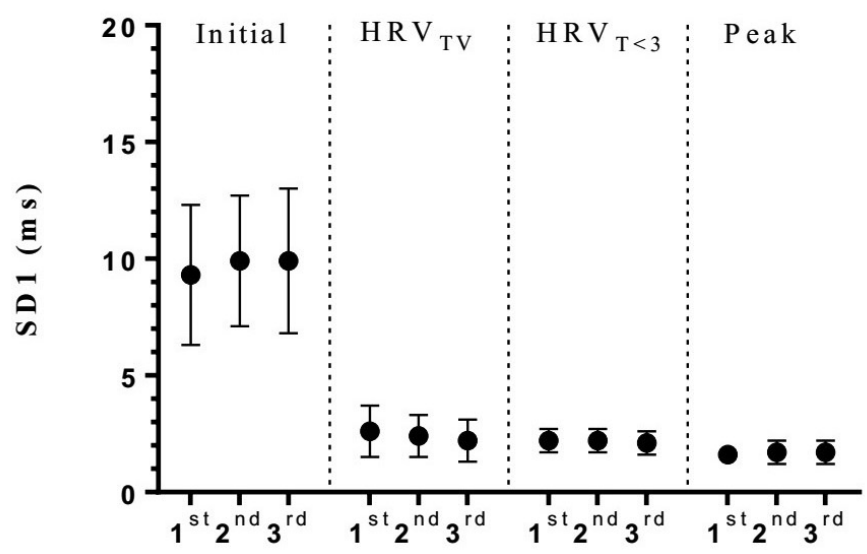

b)

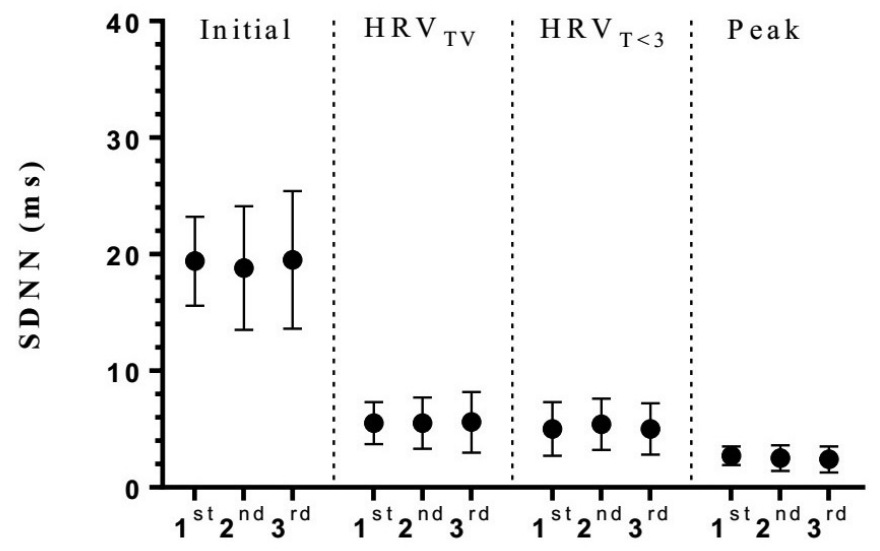

d)

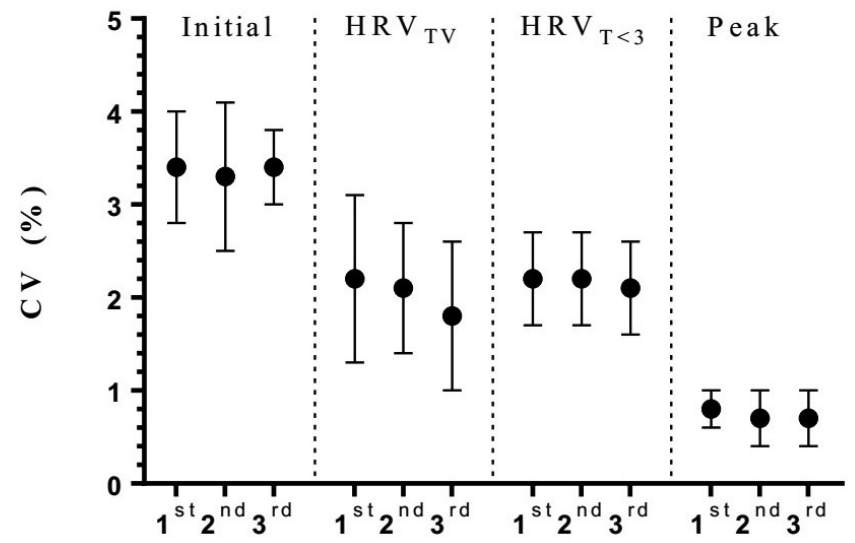

Figure 2. Heart rate and heart rate variability (SDNN, SD1 and CV) analysis at first stage of incremental exercise test (Initial), in the load corresponding to heart rate variability threshold assessed by visual method (HRVtv), mathematical method (HRVT<3) and in the last stage of incremental exercise test (Peak). *ANOVA for repeated measures $(p<0.05)$. 

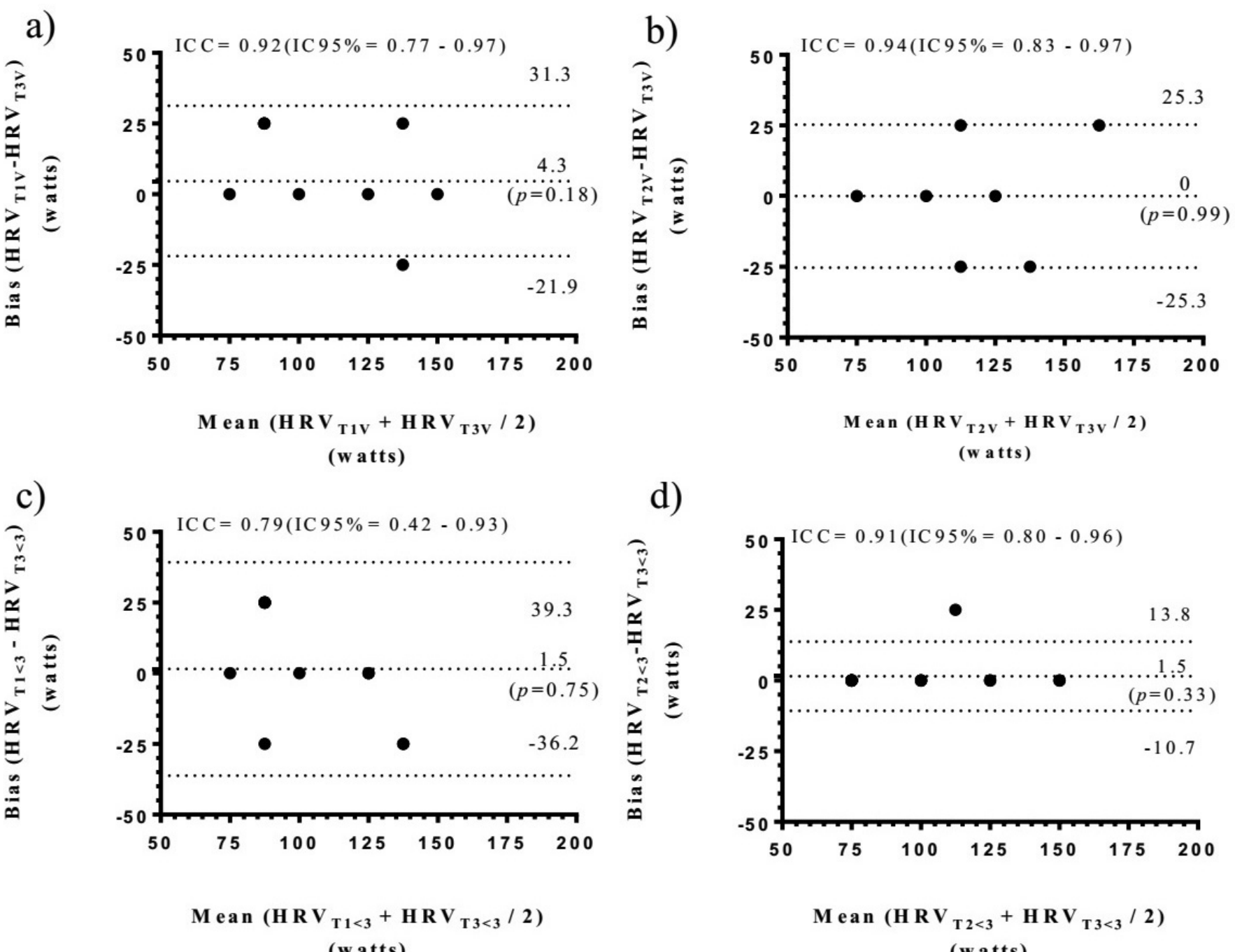

Figure 3. Bland-Altman plots and intraclass correlation coefficient (ICC) of load corresponding to heart rate variability threshold assessed by visual (A e B) and mathematical (C e D) methods using the first (A e C) and second (B e D) segment of i-RR comparatively to third segment of analysis in a three minutes stage incremental exercise test $(n=17)$.

\section{Discussion}

New and applicable findings were observed in our work, demonstrating that HRVTV can be similarly assessed by the first (HRV T1v), the second (HRV T2V) or the standard third (HRVt3v) minute segment time points of the iRR series during the IET. On the other hand, a lower ICC was observed between these different time points of analysis when the mathematical criterion (SD1 < 3ms) was used to identify the HRVT.

Despite the higher reliability and low bias observed for $\mathrm{HRV}_{\mathrm{TV} 2}$ vs HRV Tv3 comparatively to HRV $\mathrm{HV}_{\text {1 }}$ vs HRV Tv3 analyses, these differences are not important in practical or statistical context due to the high overlap of intervals of confidence in the ICC analyses. Therefore, ours results support the hypothesis that $\mathrm{HRV}_{\mathrm{T}}$ is mainly affected by fast neural mechanisms of cardiovascular regulation that can be effectively assessed by the first or second 60 s segments of $i R R$ when the visual method is used as a reference to $\mathrm{HRV}_{\mathrm{T}}$ determination.

The differences between the agreement and ICC levels observed between HRV TV and $H_{\mathrm{TV}}<3$ methods may be explained by the nature of these measurements. The visual method requires the analysis of kinetics of parasympathetic deactivation during exercise test ${ }^{27,30}$, while the mathematical method is arbitrarily defined as the first load in which the SD1 value is lower than $3 \mathrm{~ms}^{7}$. The fragility of the mathematical method may be easily visualized in the figure $1 \mathrm{~b}$, where the plateau in the parasympathetic activity can be observed at 125 watts and the $\mathrm{HRV}_{\mathrm{T}<3}$ is identified at 150 watts due to a minimal difference of $0.3 \mathrm{~ms}$. Thus, despite the validity of this approach ${ }^{16,31}$, the use of a fixed criterion of $3 \mathrm{~ms}$ to HRVT determination should be used cautiously, especially when the temporal indices of HRV (i.e. r-MSSD) are used ${ }^{31}$. 
The effect of the exercise test protocol on the submaximal and maximal variables (i.e. LT and maximal power output, respectively) has been a target of some researches ${ }^{17,32}$. Bentley, Newell and Bishop ${ }^{15}$ suggests that larger stage protocols $(>180 \mathrm{~s})$ are necessary for an appropriate determination of LT, which requires a sufficient period of time for muscular lactate to be available in the bloodstream. On the other hand, higher power output has been achieved in short stages incremental tests ${ }^{17,32}$. Thereafter, two different IET might be necessary for an optimal measure of these maximal and submaximal variables, what might be impractical either due to logistics/cost and to volunteer adherence.

Considering the $\mathrm{HRV}_{\mathrm{T}}$ assessment, the adoption of larger stage protocols have also been proposed based on the supposed necessity of a sufficient time for HR stability during the analyse ${ }^{11,12}$. In fact, our results show that HR at the $3^{\text {rd }}$ segment of analysis is slightly higher when compared to the $1^{\text {st }}$ segment both at HRVTV $(\sim 6 \mathrm{bpm})$ and at the last stage of incremental exercise test $(\sim 7 \mathrm{bpm})$. It is important to note that despite the supposition that 3-min stages are necessary for HR stabilization, this parameter has been determined without solid evidence to support this hypothesis. Thus, the effectiveness of this strategy to HR stabilization during exercise tests remains unknown.

Despite the slight instability of HR, no differences in HRV was observed at any time point of analysis during the IET, as indicated by SD1, SDNN and CV analysis. Thus, since that $\mathrm{HRV}_{\mathrm{T}}$ is determined by HRV dynamics and not by HR per se, this slight increase in HR at HRV TV3 does not appear to influence the HRV T analysis. Indeed, a high agreement between VT and $\mathrm{HRV}_{\mathrm{T}}$ has been observed even when stages with 60 seconds of duration are adopted ${ }^{31,33}$, suggesting that large stages for HRV determination may be unnecessary.

Taken altogether, our findings could be a useful tool for both clinical and sports setting, reducing 3-fold the time to complete the exercise test comparatively to conventional protocols normally used to determine HRV . Additionally, since higher maximal power output can be reached in short $(60 \mathrm{~s})$ comparatively with large $(180 \mathrm{~s})$ stage protocols ${ }^{17,32}$, the possibility of $\mathrm{HRV}_{\mathrm{T}}$ analyses by a $60 \mathrm{~s}$ stage incremental test allows an optimal measurement of these maximal and submaximal variables at the same test.

To the best of our knowledge, this is the first study to present evidence-based information showing that a 1 or 2-min stage protocol during IET could be as reliable as the traditional and longer 3-min stage for HRV T assessment. Of note, although the use of HRVT has been largely recommended for anaerobic threshold determination ${ }^{9,12,16,33}$, several questions remain obscure and require further investigations. Amongst them we can highlight: 1) is this technique valid in women? 2) what is the impact of the ergometer type on $\mathrm{HRV}_{\mathrm{T}}$ ? 3) is this technique also valid and reproducible on non-laboratorial conditions? The answers to these questions go beyond the objective of our study but are absolutely necessary both for better understand the HRVT phenomenon and for its proper use in exercise physiology and other health-related fields.

The main limitations of this study are the small number of participants and the absence of LT or VT analysis during IET. However, the validity of the HRV for predicting the load corresponding to LT or VT was not the objective of the present study and has already been tested previously ${ }^{8-12,16,33}$. Additionally, the analysis of 3 different iRR segments using the same IET to $\mathrm{HRV}_{\mathrm{T}}$ assessment may seem an inappropriate approach since the effects of different incremental protocols on the physiological and mechanical variables (i.e., Vo2 maximum and power output) are generally evaluated in different tests and/or days ${ }^{34,35}$. However, the objective of the present study was to evaluate the HRV stability over each 3-min iRR segment during IET, which cannot be properly assessed by iRR segments obtained in different exercise tests, mainly due to the interday variation of this measure $^{11}$. In this sense, due to possible differences between HR and HRV kinetics during short and long-stages incremental exercise protocols, future studies investigating the effect of different incremental protocols on validity and reliability of $\mathrm{HRV}_{\mathrm{T}}$ are necessary.

\section{Conclusion}

We concluded that HRVT can be similarly assessed by the first, the second or the traditional third $60 \mathrm{~s}$ iRR segment of each 3-min stage during an incremental exercise test, mainly when a visual method is used to identify the parasympathetic depression.

\section{References}

1. Ferreira MJ, Zanesco CL. Heart rate variability as important approach for assessment autonomic modulation. Motriz J. Phys Ed. 2016;22(2):3-8.

2. Shaffer F, McCraty R, Zerr CL. A healthy heart is not a metronome: an integrative review of the heart's anatomy and heart rate variability. Front Psychol. 2014;5(1): 1-19.

3. Araújo JA, Tricot GK, Arsa G, Queiroz MG, Santos KM, Dias ARL, et al. Blood pressure and cardiac autonomic modulation at rest, during exercise and recovery time in the young overweight. Motriz J. Phys Ed. 2016;22(1):27-34.

4. Cunha AS. Impacts of low or vigorous levels of physical activity on body composition, hemodynamics and autonomic modulation in Down syndrome subjects. Motriz J. Phys Ed. 2018;24(1):1-7.

5. Tulppo MP, Makikallio TH, Takala TE, Seppanen T, Huikuri HV. Quantitative beat-to-beat analysis of heart rate dynamics during exercise. Am J Physiol. 1996;271(1):244-252.

6. Goldberger JJ, Le FK, Lahiri M, Kannankeril PJ, Ng J, Kadish AH. Assessment of parasympathetic reactivation after exercise. Am J Physiol Heart Circ Physiol. 2006;290(6): 2446-2452.

7. Lima JRP, Kiss MAPDM. Limiar de variabilidade da frequência cardíaca. Rev Bras Ativ Saúde. 1999;4(1):29-38.

8. Gomes CJ, Molina GE. Utilização da variabilidade da frequência cardíaca para a identificação do limiar anaeróbio. Uma revisão sistemática. Rev Ed Física. 2014;25(4):675-683.

9. Vasconcellos F, Seabra A, Montenegro R, Cunha F, Bouskela E, Farinatti P. Can heart rate variability be used to estimate gas exchange threshold in obese adolescents? Int J Sports Med. 2015;36(8):654-660. 
10. Leprêtre P, Bulvestre M, Ghannem M, Ahmaidi S, Weissland T, Lopes P. Determination of ventilatory threshold using heart rate variability in patients with heart failure Surgery. 2013;12(1):2-6.

11. Karapetian GK, Engels HJ, Gretebeck KA, Gretebeck RJ. Effect of caffeine on LT, VT and HRVT. Int J Sports Med. 2012;33(7):507-513.

12. Karapetian GK, Engels HJ, Gretebeck RJ. Use of heart rate variability to estimate LT and VT. Int J Sports Med. 2008;29(8):652-657.

13. Cruz CJG, Rolim PS, Pires DS, Mendes CMO, de Paula GM, Porto LGG, et al. Reliability of heart rate variability threshold and parasympathetic reactivation after a submaximal exercise test. Motriz J. Phys Ed. 2017;23(1):65-70.

14. Faude O, Kindermann W, Meyer T. Lactate threshold concepts: how valid are they? Sports Med. 2009;39(6):469-490.

15. Bentley DJ, Newell J, Bishop D. Incremental exercise test design and analysis: implications for performance diagnostics in endurance athletes. Sports Med. 2007;37(7):575-586.

16. Sales MM, Campbell CS, Morais PK, Ernesto C, Soares-Caldeira LF, Russo $\mathrm{P}$, et al. Noninvasive method to estimate anaerobic threshold in individuals with type 2 diabetes. Diabetol Metab Syndr. 2011;3(1):1-8.

17. Bentley DJ, McNaughton LR. Comparison of W(peak), VO2(peak) and the ventilation threshold from two different incremental exercise tests: relationship to endurance performance. J Sci Med Sport. 2003;6(4):422-435.

18. Novelli FI, de Araujo JA, Tolazzi GJ, Tricot GK, Arsa G, Cambri LT. Reproducibility of Heart Rate Variability Threshold in Untrained Individuals. Int J Sports Med. 2019;40(2):95-99.

19. Tulppo MP, Hautala AJ, Makikallio TH, Laukkanen RT, Nissila S, Hughson RL, et al. Effects of aerobic training on heart rate dynamics in sedentary subjects. J Appl Physiol. 2003;95(1):364-372.

20. Miyagi R, Sasawaki Y, Shiotani H. The influence of short-term sedentary behavior on circadian rhythm of heart rate and heart rate variability. Chronobiol Int. 2019;36(3):374-380.

21. Craig CL, Marshall AL, Sjostrom M, Bauman AE, Booth ML, Ainsworth BE, et al. International physical activity questionnaire: 12-country reliability and validity. Med Sci Sports Exerc. 2003;35(8):1381-1395.

22. Tanaka H, Monahan KD, Seals DR. Age-predicted maximal heart rate revisited. J Am Coll Cardiol. 2001;37(1):153-156.

23. Giles D, Draper N, Neil W. Validity of the Polar V800 heart rate monitor to measure RR intervals at rest. Eur J Appl Physiol. 2016;116(3):563-571.

24. Porto LG, Junqueira LF, Jr. Comparison of time-domain short-term heart interval variability analysis using a wrist-worn heart rate monitor and the conventional electrocardiogram. Pacing Clin Electrophysiol. 2009;32(1):43-51.

25. Caminal P, Sola F, Gomis P, Guasch E, Perera A, Soriano N, et al. Validity of the Polar V800 monitor for measuring heart rate variability in mountain running route conditions. Eur J Appl Physiol. 2018;118(3):669-677.

26. Tarvainen MP, Niskanen JP, Lipponen JA, Ranta-Aho PO, Karjalainen PA. Kubios HRV-heart rate variability analysis software. Comput Methods Programs Biomed. 2014;113(1):210-220.

27. Candido N, Okuno NM, da Silva CC, Machado FA, Nakamura FY. Reliability of the Heart Rate Variability Threshold using Visual Inspection and Dmax Methods. Int J Sports Med. 2015;36(13)1076-1080.
28. Bland JM, Altman DG. Measuring agreement in method comparison studies. Stat Methods Med Res. 1999;8(2):135-160.

29. Hopkins WG. Measures of reliability in sports medicine and science. Sports Med. 2000;30(1):1-15.

30. Rolim PS, Matos RAC, Soares EMKK, Molina GE, Cruz CJG. Caffeine increases parasympathetic reactivation without altering resting and exercise cardiac parasympathetic modulation: A balanced placebo design. Eur J Sport Sci. 2018;19(4):490-498.

31. Queiroz MG, Arsa G, Rezende DA, Sousa LCJL, Oliveira FR, Araújo GG, et al. Heart rate variability estimates ventilatory threshold regardless body mass index in young people. Sci sports. 2018;33(1):39-46.

32. Amann M, Subudhi A, Foster C. Influence of testing protocol on ventilatory thresholds and cycling performance. Med Sci Sports Exerc. 2004;36(4):613-622.

33. Ramos-Campo DJ, Rubio-Arias JA, Ávila-Gandía V, Marín-Pagán C, Luque A, Alcaraz PE. Heart rate variability to assess ventilatory thresholds in professional basketball players. J Sport Health Sci. 2016;6(4):468-473.

34. Riboli A, Rampichini S, Ce E, Limonta E, Coratella G, Esposito F. Effect of ramp slope on different methods to determine lactate threshold in semi-professional soccer players. Res Sports Med. 2018: 1-13. Doi: 10.1080/15438627.2018.

35. Peserico CS, Zagatto AM, Machado FA. Evaluation of the Best-designed Graded Exercise Test to Assess Peak Treadmill Speed. Int J Sports Med. 2015;36(9):729-734.

\section{Acknowledgements}

The authors would like to thank all participants of the study, the laboratory technicians and the Centro Universitário Euro Americano-UNIEURO for financial support (001/2017).

\section{Corresponding author}

Carlos Janssen Gomes da Cruz

Group of studies and research in cardiac autonomic function, department of physical education, Centro Universitário Euro Americano-UNIEURO, Brasília-Brazil. Avenida das Nações, Trecho 0, Conjunto 05, Brasília, Brazil

Email: carlos.gomes@unieuro.com.bror janssengomes@gmail.com

Manuscript received on December 18, 2018

Manuscript accepted on June 12, 2019

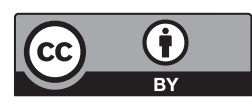

Motriz. The Journal of Physical Education. UNESP. Rio Claro, SP, Brazil - eISSN: 1980-6574 - under a license Creative Commons - Version 4.0 